\title{
Microwave-Based SAR Technique for Pipeline Inspection using Autofocus Range-Doppler Algorithm
}

\author{
Mohammed D. Buhari, Student, IEEE, Gui Yun Tian, Senior Member, IEEE, and Rajesh Tiwari, Member, IEEE
}

\begin{abstract}
In non-destructive testing and evaluation, microwave-based synthetic aperture radar (SAR) imaging have shown great potential in the detection of defects on buried objects such as pipes. However, due to pipe curvature and high standoff distance when inspecting an insulated pipe, the useful defect information used to characterise the pipe image is lost as a result of low signal-to-noise ratio (SNR) resulting in a blurred and unfocused image. In this paper, we proposed a robust microwave-based SAR imaging using autofocus range-Doppler algorithm (RDA) for the inspection of an insulated pipe. Singular value decomposition (SVD) is used to mitigate the effect of the insulation layer by removing dominant singular values that characterise the insulation layer, and the autofocus RDA is designed to refocus the SAR image using RDA residual refocusing. SNR, improvement factor (IF) and squared error (SE) are used to evaluate the qualitative image information of the defect on the pipe. Experimental results showed the efficacy of the method in detecting defects on an insulated pipe, in particular, a significant reduction in the noise content of the image compared to the known SAR Omega-k algorithm. It was found that the autofocus RDA gave higher values of SNR and IF $(3 \mathrm{~dB}$ and $6 \mathrm{~dB})$ compared to the Omega-k algorithm (-1 dB and $2 \mathrm{~dB}$ ) respectively.
\end{abstract}

Index Terms-Autofocus RDA, image reconstruction, improvement factor, pipeline inspection, signal-to-noise ratio (SNR), singular value decomposition (SVD), Synthetic Aperture Radar (SAR).

\section{INTRODUCTION}

$\mathbf{I}$ $\mathrm{N}$ the transportation of oil and natural gas, pipelines are the most efficient and economical way used to transport fluid across the globe. It provides an alternative transportation system that is easier and more effective than the conventional methods of using vehicles [1]. However, as the number of pipelines increases, the pipes become vulnerable to attack by both internal and external defects, third-party damage and manufacturing flaws especially when the pipes are either insulated or buried underground [2]. This may lead to leakages threatening the development of the national economy, lives and properties. To forestall the occurrence of these leakages, regular inspections of pipelines are needed to ensure the health and safety of the pipes. These inspections are normally carried out using non-destructive testing and evaluation (NDT\&E) [3]. In the area of NDT\&E, a range of inspection methods are available for pipeline integrity that include ultrasonic technique [4] [5], pulsed eddy current [6] [7], magnetic flux leakage

M. D. Buhari and G. Y. Tian are with the Department of Electrical and Electronic Engineering, Newcastle University, Newcastle Upon Tyne, NE1 7RU, and R. Tiwari is with NSL Nottingham.
(MFL) [8] [9], and electromagnetic acoustic transducers [10]. While tremendous progress has been made on mainline piping systems, which are conventionally piggable, a key difficulty facing pipeline integrity personnel has been the ability to inspect and gather data on pipelines that are unpiggable or hard to navigate due to the smaller size of the pipes usually installed in the cities. Inspection tools must navigate these lines and accurately measure the size and specific location of all defects. In such areas, surface inspection of the pipes is employed. Microwave NDT\&E technique is one of the popular methods for pipeline surface inspection using methods such as ground penetration radar (GPR) [11], synthetic aperture radar (SAR) [12]. They are non-contact inspection methods and have since become hotspots in the pipeline industry because of its simplicity, high detection efficiency and most of them do not require an excitation source [13].

For the inspection of an insulated pipe, the pipe curvature and high standoff distance are among the major challenges that need to be mitigated; otherwise, the defect information is lost making the pipe image to be blurred and unfocused. Regarding the pipe curvature, a robust technique is needed for the inspection as the surface of the insulated pipe is no longer flat. This is because for the same inspection angle in the specimen, the full skip distance of the probes will always be bigger in a pipe than in a flat plate and it increases with a decrease in the pipe diameter. As for the insulated pipe, the additional layer increases the standoff distance making the defect characterisation difficult. Several methods were proposed to compensate for these challenges quantitatively and qualitatively [3]. Quantitative analysis uses inverse methods that are generally nonlinear to get the electrical and mechanical distribution, size, shape and location of the defect. Successive iterations are used to linearize the nonlinear inverse problem using different approximation methods such as Gauss-Newton method [14], distorted Born iteration [15], and sparsity regularisation [16]. The drawbacks of these techniques include computational time to achieve an optimised solution and the complexity of the method. In qualitative analysis, the reflectivity information is used to get a qualitative image of the defects. In most cases, the technique uses migration-based algorithms for the defect image reconstruction performed either in time domain (time shift) or frequency domain (phase shift). Microwave imaging method using chirp scaling, frequency-wavenumber $(\omega-k)$, range-Doppler algorithm (RDA), and matched filter are all qualitative imaging methods [17]. In [18], a two-dimensional microwave imaging of irregular shaped metal targets to obtain 
the full 2-D geometry of the target was proposed. The method uses the X-band frequency range for the near-field target imaging.

Despite the aforementioned developments in microwave non-destructive imaging method, there are few microwave SAR techniques used in layered media to appropriately compensate for the pipe curvature and high standoff distance introduced by the insulation layer. In [11], a robust adaptive detection method for buried pipes using GPR was proposed to enhance the signal of interest and reduces the layer contribution. This reduces the probability of false arm (PFA). In [19], a new microwave radar imaging prototype has been developed for inspecting wood material. It is based on a set of custom antennas positioned in proximity to the sample, and a hardware design to reconstruct tomographic maps of a wooden slab or a tree trunk. The data inversion has been obtained by using a two-step procedure combining a qualitative approach, with a quantitative approach based on an inexact-Newton method. In [3], a robust method was proposed to compensate for the electrical, physical and inhomogeneous properties of the layer using two approaches of piecewise SAR (PW-SAR) and Wiener filter-based SAR (WL-SAR). PW-SAR was used to account for the electrical and physical properties of the layer while WL-SAR was used to account for multiple reflections and signal attenuation of the electromagnetic signal as a result of the inhomogeneity of the layer. Both approaches use the $\omega-k$ algorithm and the targeted application was to detect rebar embedded in a layered media. The approaches imaged the rebar showing the worn out areas. However, when tiny defects/cracks need to be detected, $\omega-k$ algorithm as used in [3] may not be a good choice because the Stolt mapping (SM) interpolation used in $\omega-k$ algorithm amplifies the noise content and the small motion error in the raw data and this brings an extra RCM-error [20]. Also, the sample used in [3] is rebar and therefore, the method does not consider the curvature and defect shape which need to be considered in pipeline inspection.

To address the problems of $\omega-k$ algorithm's limitation, this paper proposed a new approach using SVD-autofocus RDA SAR-based microwave imaging for pipeline inspection under an insulation media. SVD has been used previously in GPR to improve the SNR of the SAR images by decomposing the image into several Eigen-images [21]. It has also been used in through-the-wall radar imaging (TWRI) to remove the wall clutter and to detect behind-the-wall targets from Bscan images [22]. In [23], an SVD-based method was used to mitigate the wall clutter in the image based on the fact that the wall reflections reside in the first Eigen-images and the target reflections span several Eigen-images. Based on the insulation layer structure and the curvature characteristics of the pipe, singular value decomposition (SVD) is used to suppress the effect of the insulation layer, and autofocus range-Doppler algorithm (RDA) is designed to refocus the SAR image. The method proves to be robust for inspecting insulated pipe to reveal the qualitative image of the defect shape and location. This is because the RDA residual refocusing ensures that the inherent noise in the raw data is compensated, eliminates the effect of the pipe curvature and not amplified as with the

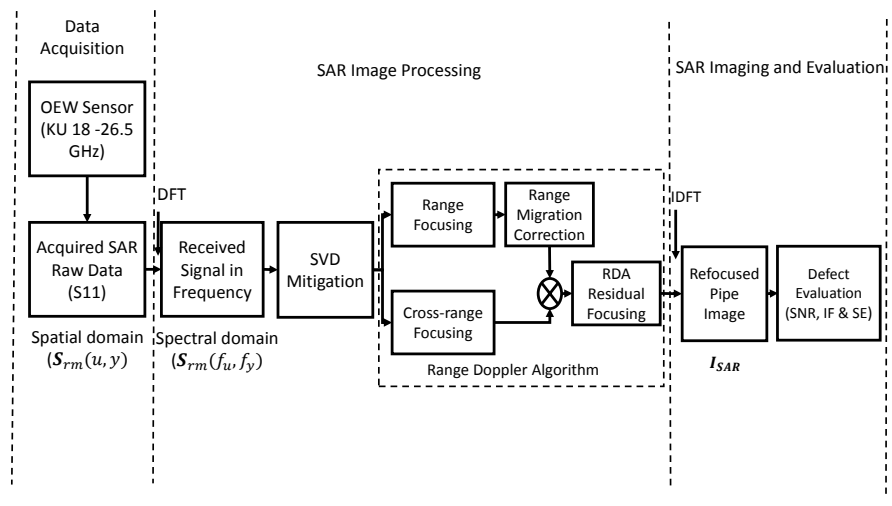

Fig. 1. Block diagram microwave SAR imaging for pipeline inspection using autofocus range-Doppler algorithm.

case of SM interpolation used in the $\omega-k$ algorithm. The method also reduces the system complexity as well as the processing time of the reconstructed pipe image compared to other approaches such as the ones used in [3].

The remaining part of the paper is organized as follows: Section II describes the system and methods for autofocus RDA SAR-based pipeline inspection. In Section III, the results and discussion are presented based on experimental validation of the approach. The conclusion and future work are presented in Section IV.

\section{System And Methods For Autofocus RDA SAR-BASED PIPELINE INSPECTION}

This section introduces the microwave-based SAR system configuration, the influence of the insulation layer, and the SVD-RDA method to reconstruct the image of the insulated pipe. The SVD is used to mitigate the effect of the insulation layer, and autofocus RDA is designed to refocus the SAR image using RDA residual refocusing.

\section{A. Design of Microwave NDT based SAR System}

The design of the microwave-based SAR system using autofocus RDA follows the procedure shown in Fig. 1. It started with the raw data acquisition from the vector network analyser (VNA) in spatial domain. The sensor used is an open-ended waveguide (OEW) operating at X-band, Ku-band and K-band. For the signal processing, the received signal is converted to frequency domain (spectral domain). SVD is used to suppress or mitigate the effect of the insulation layer, and the output of the SVD is then refocused using RDA. The combination of SVD-RDA is termed autofocus RDA because the focusing is automatically done in these two processes. The SAR image is formed by taking the product of the range profile with the corresponding cross-range profile. To evaluate the performance of the technique against other known SAR techniques, the SNR, improvement factor (IF) and square error are used to show the strength of the autofocus RDA in dealing with the pipe curvature and high standoff distance.

For the microwave SAR system configuration, the choice for the operational mode of the SAR geometry depends on the targeted application [24]. In our previous work in [12], broadside target was developed for a point target on a flat surface as shown in Fig. 2 (a). In the model, the SAR system moves along the cross-range axis (u-axis) from position $-L$ 


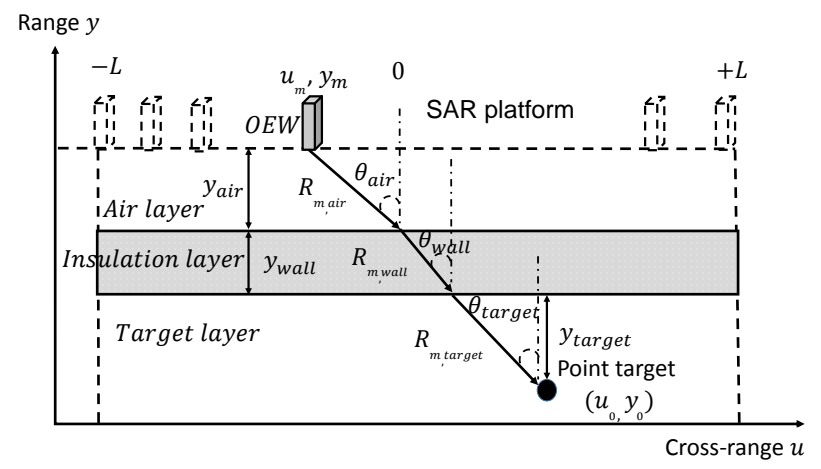

(a)

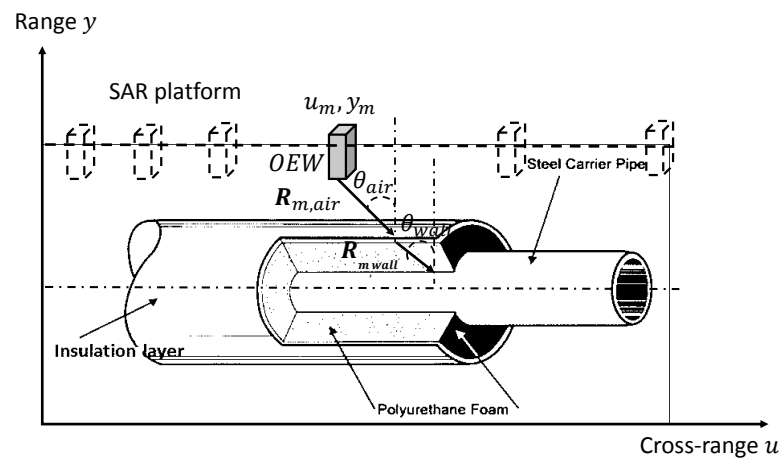

(b)

Fig. 2. Microwave SAR system configuration (a) flat surface, (b) curved surface.

to $+L$. The cross-range axis is parallel to the ground along the range axis (y-axis), and the target is buried under an insulation layer. An OEW is used as the transmit/receive antenna to transmits the signal $s(t)$ towards the target at a position of $\left(u_{0}, y_{0}\right)$. The signal gets refracted/reflected by the insulation layer while the remaining part of the signal penetrates through it reaching the target. The signal is then reflected by the target characterised by the target reflectivity coefficient $\alpha_{m}$. The insulation layer is assumed to be homogeneous with uniform dielectric constant, and the signal is transmitted perpendicular to the tangent surface of the insulation layer. The received signal can be modelled using plane wave Fresnel transmission/reception coefficient obtained from Maxwell's equations [22]. The received signal can be expressed as

$$
\begin{aligned}
& s_{r m}(t)=\alpha_{m} s_{t}\left(t-\tau_{m}(u, y)\right) \\
s_{r m}(t)= & \alpha_{m} x_{r}\left(t_{y}-\tau_{m}(u, y)\right) e^{j \pi K_{r}\left(t_{y}-\tau_{m}(u, y)\right)^{2}} \\
& x_{u}\left(t_{u}-\frac{u_{0}}{V}\right) e^{\frac{-j 4 \pi}{\lambda} \tau_{m}(u, y)}
\end{aligned}
$$

where $\alpha_{m}$ is the reflectivity coefficient, $s_{t}$ is expressed in terms of the envelopes of the transmitted signal in range $\left(x_{r}().\right)$ and cross-range axis $\left(x_{u}().\right), V$ is the radar radial velocity, $K_{r}$ is the range wave number, $\lambda$ is the wavelength and, $\tau_{m}\left(u_{m}, y_{m}\right)$ is the time delay between the transmitter to the target and back at the receiver and is given by

$$
\tau_{m}(u, y)=\frac{2 R_{m, a i r}}{c}+\frac{2 \sqrt{\epsilon} R_{m, \text { wall }}}{c}+\frac{2 R_{m, \text { target }}}{c}
$$

where $R_{m, a i r}, R_{m, \text { wall }}$ and $R_{m, \text { target }}$ are the range with respect to air, wall and the target, $\epsilon$ is the dielectric constant of the insulation layer and $\mathrm{c}$ is the speed of light.

The distances $R_{m, a i r}, R_{m, \text { wall }}$ and $R_{m, \text { target }}$ can be estimated using

$$
\left.\begin{array}{rl}
R_{m, \text { air }} & =\frac{y_{\text {air }}}{\cos \left(\theta_{\text {air }}\left(u_{0}, y_{0}\right)\right)} \\
R_{m, \text { wall }} & =\frac{y_{\text {wall }}}{\cos \left(\theta_{\text {wall }}\left(u_{0}, y_{0}\right)\right)} \\
R_{m, \text { target }} & =\frac{y_{\text {target }}}{\cos \left(\theta_{\text {target }}\left(u_{0}, y_{0}\right)\right)}
\end{array}\right\}
$$

where $y_{\text {air }}$ is the standoff distance between the antenna and the insulation layer, $y_{\text {wall }}$ is the thickness of the insulation layer and $y_{\text {target }}$ is the distance between the wall and the target.

The reflection coefficient $\alpha_{m}$ taken into account the dielectric constant is given by [22]

$$
\alpha_{m}=\frac{\rho\left(1-e^{-2 j \sqrt{\epsilon} k_{m} y_{\text {wall }}}\right)}{1-\rho^{2} 1-e^{-2 j \sqrt{\epsilon} k_{m} y_{w a l l}}}
$$

where $k_{m}=w_{m} / c$ defined the wave number and $\rho$ is the local Fresnel reflectivity coefficient given by

$$
\rho=\frac{1-\sqrt{\epsilon}}{1+\sqrt{\epsilon}}
$$

In the case of pipeline inspection where the layer is curved and not flat as shown in Fig. 2(b), the derivation for the model thus far has to be modified. The surface (i.e. the insulation layer) is curved along the pipe and we assumed that there is no any layer between the insulation and the pipe (target); hence, $y_{\text {target }}$ becomes zero. The same modelling used for the flat surface can then be applied since $R_{m, a i r}$ and $R_{m, \text { wall }}$ are unique for each position. The only variables that are affected are the $y_{\text {air }}$ and $y_{\text {wall }}$ which will be compensated as will be shown later. The same formulation therefore holds and the target layer is eliminated. Thus, for the signal modelling of the curved surface, the following relationships are obtained:

$$
\left.\begin{array}{c}
\tau_{m}(u, y)=\frac{2 R_{m, a i r}}{c}+\frac{2 R_{m, \text { wall }}}{c} \\
R_{m, \text { air }}=\frac{y_{\text {air }}}{\cos \left(\theta_{\text {air }}\left(u_{0}, y_{0}\right)\right)} \\
R_{m, \text { wall }}=\frac{y_{\text {wall }}}{\cos \left(\theta_{\text {wall }}\left(u_{0}, y_{0}\right)\right)}
\end{array}\right\}
$$

The received signal is (2) is then converted to frequency domain using discrete Fourier transform (DFT) at a given SAR position $m$ using

$$
\begin{aligned}
\mathbf{S}_{r m}\left(f_{u}, f_{y}\right)= & \alpha_{m} \mathbf{X}_{r}\left(\frac{f_{r}}{K_{r}}\right) \mathbf{X}_{u}\left(\frac{f_{r}-f_{d c}}{K_{u}}\right) \\
& e^{-j \pi\left(\frac{f_{r}^{2}}{K_{r}}+\frac{2 f_{u}\left(u_{0}+u_{c}\right)}{V} \frac{4 \pi R_{m}}{\lambda} \Theta\left(f_{u}, f_{y}\right)\right)}
\end{aligned}
$$

where $f_{u}$ is the cross-range Doppler frequency, $f_{r}$ is the range frequency, $f_{d c}$ is the Doppler centroid frequency, $R_{m}$ is the superposition of $R_{m, a i r}$ and $R_{m, \text { wall }}, K_{u}$ is the cross-range 
FM rate and $\Theta\left(f_{u}, f_{y}\right)$ is the phase of a single sinusoid given by

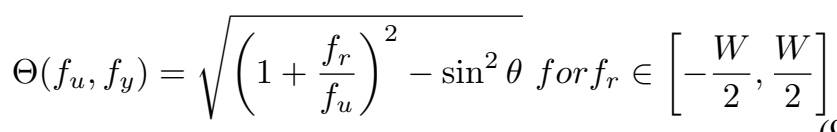

where $W$ is the bandwidth of the signal and $\theta$ is the squint angle.

To image the pipe under an insulating layer, a stepped frequency signal is utilised by transmitting monochromatic signal with frequencies equispaced based on the available bandwidth defined by

$\omega_{m}=\omega_{0}+\left(\omega_{0}+\Delta \omega\right)+\ldots+\left(\omega_{0}+m \Delta \omega\right)$ for $m=1, \ldots M$

where $\omega_{0}$ is the start frequency, $\Delta \omega$ is the step size and $\left(\omega_{0}+\right.$ $m \Delta \omega)$ is the stop frequency.

Different approaches exist that can be used to form the image of the pipe such as back-projection algorithm [25], $\omega-k$ algorithm [3], beamforming [26], compressed sensing [27], and MUSIC-LSE [12]. However, as we are operating within the near-field region where a tiny defect needs to be detected on the reconstructed pipe image under insulation, the acquired SAR raw data need to be pre-processed and refocused before the reconstructing the pipe image. This requires a robust signal processing approach to deal with the curvature of the pipe and high standoff distance introduced by the insulation layer resulting in signal attenuation. The insulation layer can be suppressed using SVD by first identifying the insulation and the pipe signal subspaces. It then projects the SAR signal onto subspace that is orthogonal to the insulation subspace. RDA is then used to refocus the resultant data from SVD using RDA residual refocusing, and its effectiveness will be shown later. At first, the detection problem due to pipe curvature, high standoff distance, and the insulation layer are discussed in the next section followed by the proposed solution.

\section{B. Influence of Pipe Insulation Layer and SVD Mitigation}

In this study, a steel pipe sample of length $600 \mathrm{~mm}$ and diameter of $50 \mathrm{~mm}$ is insulated using a Climaflex Branded closed-cell Polyethylene with a thickness of $40 \mathrm{~mm}$ curved around the pipe. Three types of defect orientation are considered namely vertical, horizontal and circular. Detecting these kinds of defects on an insulated pipe pose challenges during the inspection. This is because the insulation layer introduces an additional standoff distance coupled with the curvature of the surface, thereby attenuating the transmitted signal before reaching the pipe. As a result, the useful information used to characterise the defect on the pipe are lost making the image to be blurred and unfocused. To address this challenge, the insulated pipe is decomposed into two subspaces namely the insulation subspace and the pipe subspace using their eigenvalues based on SVD. SVD is a valuable tool used in signal processing to analyse the image quality and the independent information that characterise the unknown components in the presence of noise [28]. This is done by finding the optimal matrix decomposition in a least square sense which groups the maximum signal energy into singular values according to their energies [29]. The SVD projection enables us to know the singular values that belong to the insulation subspace and those that belong to the pipe subspace. Using these features, the insulation layer effect can be suppressed/mitigated.

To apply SVD on the acquired SAR raw data, the received signal in (10) is rearranged in matrix form to get (full details can be found in our previous work in [30]- [32])

$$
\mathbf{S}_{r m}=\alpha_{m} \mathbf{\Upsilon} \boldsymbol{\Phi}+N_{N}
$$

where $N_{N}$ is the additive white Gaussian noise (AWGN), $\mathcal{N} \sim$ $\left(0, \sigma_{\omega}^{2}\right)$, and

$$
\begin{gathered}
\boldsymbol{\Phi}=\left[\begin{array}{c}
e^{-j \pi\left(\frac{2 R m}{c-V} f_{u} f_{y}-1\right)} \\
\vdots \\
e^{-j 2 N \pi\left(\frac{2 R m}{c-V} f_{u} f_{y}-1\right)}
\end{array}\right] \\
\mathbf{\Upsilon}=\left[\begin{array}{ccc}
\left(Q_{k, r} S(r+1)\right)_{1,1} & \cdots & \left(Q_{k, r} S(r+1)\right)_{1,2 N} \\
\vdots & \ddots & \vdots \\
\left(Q_{k, r} S(r+1)\right)_{N, 1} & \cdots & \left(Q_{k, r} S(r+1)\right)_{N, 2 N}
\end{array}\right] \\
Q_{k, r}=\left\{\begin{array}{ccc}
-\frac{1}{2} j \tan \left(\frac{\pi(k+r)}{2}\right), & \text { for } & k=r \\
\frac{1}{2} j \frac{1}{\tan \left(-\frac{\pi(k-r)}{2}\right)}, & \text { for } & k \neq r \\
-\frac{j}{2}\left[\tan \left(-\frac{\pi(k-r)}{2}\right)+\tan \left(\frac{\pi(k+r)}{2}\right)\right] \\
\text { for } \quad k+r=\text { even } \\
\frac{j}{2}\left[\frac{1}{\tan \left(\frac{-\pi(k-r)}{2}\right)}+\frac{1}{\tan \left(\frac{\pi(k+r)}{2}\right)}\right] \\
\text { for } k+r=\text { odd }
\end{array}\right.
\end{gathered}
$$

For the given received signal in (11) $\mathbf{S}_{r m} \in R^{m n}$ with $m>n$, the SVD of $\mathbf{S}_{r m}$ can be found by decomposing the received signal into subspaces given by

$$
\mathbf{S}_{r m}=U \sum V^{H}=\sum_{i=1}^{n} u_{i} \sigma_{i} v_{i}^{H}
$$

where $U=\left(u_{1}, u_{2}, \ldots u_{n}\right)$ and $V=\left(v_{1}, v_{2}, \ldots v_{m}\right)$ are the left and right singular vectors of $\mathbf{S}_{r m}, \sum=$ $\operatorname{diag}\left(\sigma_{1}, \sigma_{2}, \ldots, \sigma_{n}\right)$ is a rectangular matrix that contains the singular values of $\mathbf{S}_{r m}$ arranged in the form $\sigma_{1} \geqslant \sigma_{2} \geqslant \ldots \geqslant$ $\left.\sigma_{n} \geqslant 0\right)$, and $H$ is the Hermitian transpose.

Equation (15) decomposes the received signal into its singular values and singular vectors. The insulation subspace is spanned by the first singular vector that is associated with a given singular value, while the remaining singular values are considered as subspace distortion. This subspace distortion is characterised by a distortion index as defined in [22] and it is adopted in this paper. Using the distortion index, the effect of the insulation can be eliminated. However, despite mitigating the effect of the insulation layer using the singular values of the SVD, the reconstructed image pipe is unfocused making the image to be blurred mainly because the signal is attenuated (noisy) at high standoff distance and the curvature nature of the pipe. To address this problem, autofocus RDA is used to focus the resultant raw data after SVD before reconstructing 
the image of the pipe. The approach is discussed in the next section.

\section{Autofocus RDA SAR-Based Image Processing}

RDA is one of the most widely used algorithms in SAR data processing to obtain high-resolution images. This is due to its simplicity and can accommodate different range parameters independent of the radar transmitted signal [33]. In this study, RDA is adopted because of its accuracy/efficiency/generality trade-offs compared to other existing SAR algorithms. In comparison to the $\omega-k$ algorithm, when tiny defects/cracks need to be detected, the SM used in $\omega-k$ algorithm amplifies the small motion error bringing an additional RCM-error in the image. However, RDA does not suffer from these problems although, it is relatively slow compared to the $\omega-k$ algorithm and cannot be used when large squint angles are involved. This is not a problem for our case since the inspection is done in the near-field region. Three main stages are used in the implementation of the RDA namely range compression and/or focusing, range cell migration (RCMC) and azimuth or crossrange focusing. In the range compression, the property similar to pulse compression is used i.e. $h(t) * h^{*}(-t)$. For the SAR raw data in (2), the range compression is applied to the raw data in a similar way. In our case, the acquired SAR raw data is in the spatial domain and the range compression is performed in this domain. It is then converted to the frequency domain using DFT as shown in (8), (11) and (15). Therefore, this stage is adopted from the previous section. The next stage is the RCMC and this is done to correct the migration associated with the image as a result of the movement of the sensor which normally gives a hyperbola shape.

To perform RCMC, since the phase of the received signal largely depends on the phase of the single sinusoid as shown in (11), the phase has to be matched to refocus the image. Equation (3) can be expressed in terms of Taylor's series as

$$
\begin{aligned}
\Theta\left(f_{u}, f_{y}\right)= & \sqrt{\left(1+\frac{f_{r}}{f_{u}}\right)^{2}-\sin ^{2} \theta} \\
& \approx \cos \theta+\frac{f_{r}}{\cos \theta f_{c}}-\frac{\sin ^{2} \theta}{2 \cos ^{3} \theta f_{c}^{2}} f_{r}^{2}
\end{aligned}
$$

From (16), the third term in $f_{c}^{2}$ is what is responsible for the range migration making the pipe image curved and unfocused. To account for this migration, a frequency compensation term is introduced given by

$$
\phi_{r}=\frac{R_{m}}{c}\left(\frac{f_{u}}{f_{u, m}}\right)^{2} f_{r}
$$

Using the compensation term, the new vector of the received signal with RMC is formed as

$$
\mathbf{S}_{r m}\left(f_{u}, f_{y}\right)_{R M C}=\mathbf{S}_{r m}\left(f_{u}, f_{y}\right) \cdot e^{j 2 \pi \phi_{r}}
$$

After RCMC, the cross-range (azimuth) focusing is performed using the phase of the single sinusoid given by

$$
\phi_{c r}=\left(\frac{2 R_{m}}{\lambda} \sqrt{1-\left(\frac{f_{u}}{f_{u, m}}\right)^{2}}+\frac{u_{c}}{V} f_{u}\right)
$$

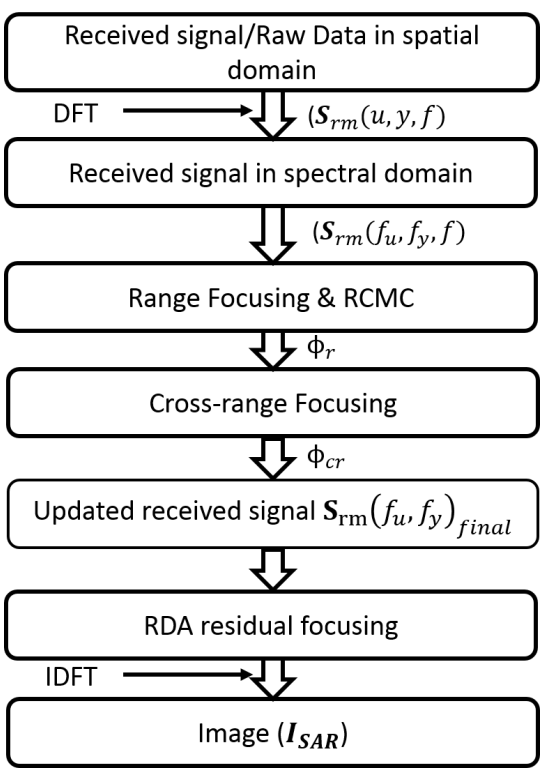

Fig. 3. Flowchart of the SAR autofocus RDA imaging.

The final received signal after completing RDA becomes

$$
\mathbf{S}_{r m}\left(f_{u}, f_{y}\right)_{\text {final }}=\mathbf{S}_{r m}\left(f_{u}, f_{y}\right) \cdot e^{j 2 \pi \phi_{r}} \cdot e^{j 2 \pi \phi_{c r}}
$$

Using (20), an RDA residual focusing is performed automatically and this ensures that any blurring contains in data is alleviated before forming the final image. This is done by using the RDA to correct any residual range curvatures and residual phase. The focused SAR image is formed by taking the IDFT of (20) to get

$$
\mathbf{I}_{S A R}=\mathcal{F}^{-1}\left\{\mathbf{S}_{r m}\left(f_{u}, f_{y}\right)\right\}
$$

The flowchart of the autofocus RDA is shown in Fig. 3 and the entire microwave SAR imaging procedure is as summarised in Fig. 1.

\section{RESUlTS AND DisCUSSION}

In this section, the proposed microwave-based SAR imaging using the autofocus RDA for pipeline inspection described in Section II is tested and validated through experiments. The set-up for the experiment is discussed with all the values and assumptions made presented. XY scanner is used to scan the insulated pipe and the raw data collected is then processed using the SVD-autofocus RDA technique. The technique is validated using performance measures of SNR, IF and SE and the results are compared with the known $\omega-k$ algorithm technique. Two set of experiments were carried out to show the effectiveness of the approach in compensating for the pipe curvature and high standoff distance introduced by the insulation layer. The technique is also extended to imaging of $3 \mathrm{D}$ pipe as this reveals more information about the defect shape and location.

\section{A. Experimental Setup and Sample Description}

To demonstrate the effectiveness of the autofocus RDA technique for pipeline inspection, an experimental study is carried out to validate the method using the experimental set-up shown in Fig. 4. The measurement set-up consists of 


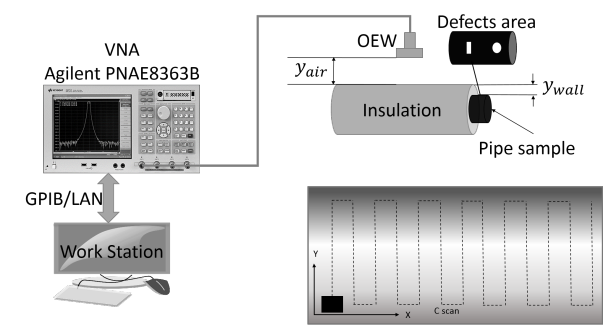

(a)

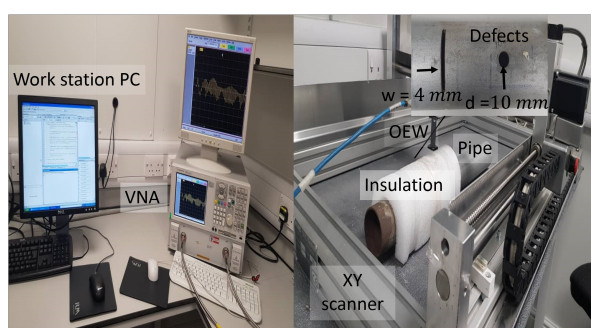

(b)

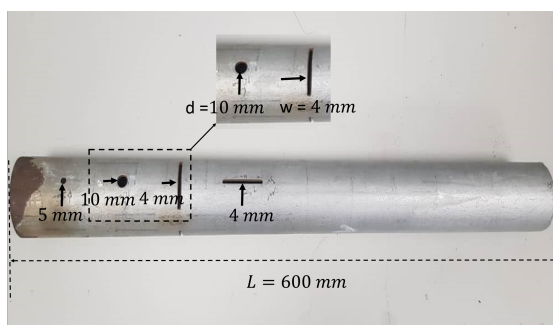

(c)

Fig. 4. Overall experimental setup (a) Schematic design. (b) Picture of experimental setup.(c) Steel pipe sample.

an E8363B VNA from Agilent Technologies with frequency range of $10 \mathrm{MHz}$ to $40 \mathrm{GHz}$, three OEWs with operating frequency ranges within X-band $(8.2-12.4 \mathrm{GHz}), \mathrm{Ku}$-band $(12.4-18 \mathrm{GHz})$ and $\mathrm{K}$-band $(18-26.5 \mathrm{GHz})$. The VNA is connected the OEW at one end via an RF cable of length 3 $\mathrm{m}$ at port one, while at the other end, it is connected to a workstation PC via a general purpose interface bus (GBIP). The OEW is attached to an XY scanner which scans over the pipe sample using $\mathrm{C}$ scanning pattern as shown in Fig. 4. The VNA uses sweep-frequency measurement to evaluate the sample from the complex signal reflection of the pipe. The SAR raw data acquired from the VNA contains the magnitude and phase of the microwave reflection coefficient known as $S_{11}$ parameter and the results obtained are analysed in MATLAB. The remaining design parameters used for the experiment are set to the following values: Number of points is 1601; scanning area $150 \mathrm{~mm} \times 150 \mathrm{~mm}$; step size of $2 \mathrm{~mm}$. The sample is made up of a steel pipe sample of length $600 \mathrm{~mm}$ and 50 $\mathrm{mm}$ in diameter. The sample has defects/cracks of different sizes and orientations. Two defects on the pipe are used, a vertical crack with a width of $4 \mathrm{~mm}$ and a circular defect with a diameter of $10 \mathrm{~mm}$. The pipe is insulated from the outer surface using an insulation layer that is made up of a Climaflex Branded closed-cell Polyethylene with a thickness of $40 \mathrm{~mm}$ curved around the pipe, with a thermal conductivity of $0.034 \mathrm{~W} / \mathrm{Mk}$ at $0^{\circ} \mathrm{C}$ and temperature range of -45 to 105 ${ }^{\circ} \mathrm{C}$ [34]. Two set of experiments were carried out one to show the effect of the pipe curvature at high standoff distance and the other to study the SVD mitigation on an insulated pipe and they are discussed in the next section.

\section{B. Influence of Pipe Curvature and High Standoff Inspection}

Using the experimental setup shown in Fig. 4, the experiment was carried out by scanning the steel pipe sample using raster scan controlled through the MATLAB programme. As explained earlier, OEW was used as the sensor because it offers a relatively wider bandwidth which is needed to achieve a finer range and cross-range resolution [35]. Three OEWs were used and the image obtained were compared to choose the best frequency range for our application. It is important to point out that OEW may present practical challenges of antenna radiation pattern, directivity, antenna-to-air-match and the pipe curvature when used for pipeline inspection. The first three challenges can be addressed using the methods described in [3] and the same approach was adopted in our work. For the pipe curvature, it is addressed through RDA residual focusing performed automatically on the raw data to focus

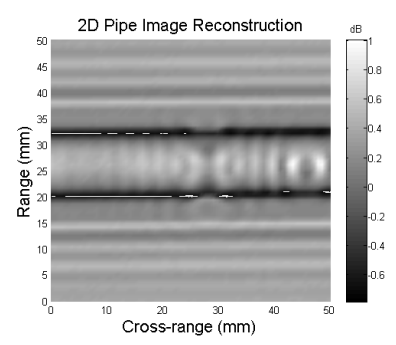

(a)

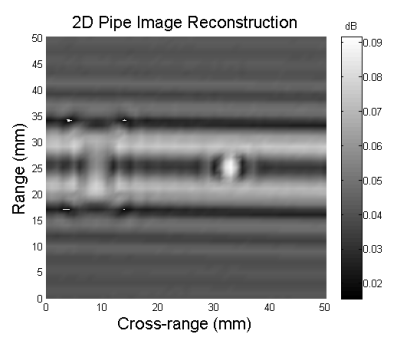

(b)

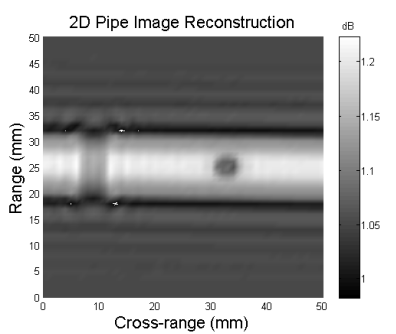

(c)

Fig. 5. Reconstructed images of the defect on the pipe (a) X-band image of the pipe, (b) Ku-band image of the pipe, (c) K-band image of the pipe.

the curvature of the pipe image. To show the performance of the autofocus RDA approach, the defect sample was scanned using the X-band, Ku-band and the K-band OEWs. The raw data in spatial domain is then collected from the complex reflection coefficients defined by the $S_{11}$ parameter calibrated based on the aperture of the waveguide. The raw data is then focused by subtracting the mean of the spatial sample from the original spatial sample. This ensures that the mismatch as a result of the high standoff distance is reduced thereby focusing the raw data collected. The resultant raw data is then processed using RDA and this addressed the challenge in dealing with the pipe curvature and at this stage, only the steel pipe was scanned without the insulation. The case of the insulated pipe and its effects on the reconstructed image will be presented later. Using the defective sample shown in Fig. 4(c), the reconstructed pipe image at a standoff distance of $40 \mathrm{~mm}$ for X-band, Ku-Band and the K-band are shown in Fig. 5. From the processed SAR image using RDA, it can be seen that both the images from the three different bands used successfully detected the defects on the pipe image. However, the K-band gives a higher-resolution image compared to the $\mathrm{X}$-band and the Ku-band and therefore, it is used for the rest of the experiment. The shape and location of the defect can also be measured from the reconstructed image. 


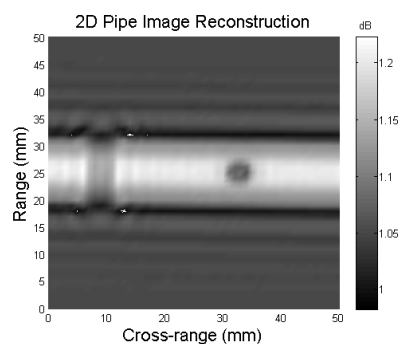

(a)

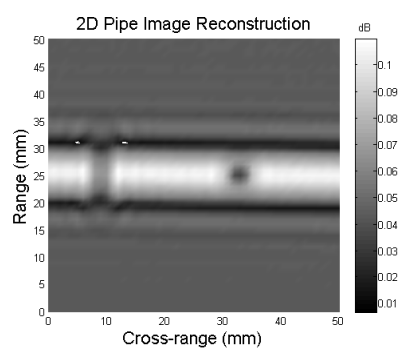

(e)

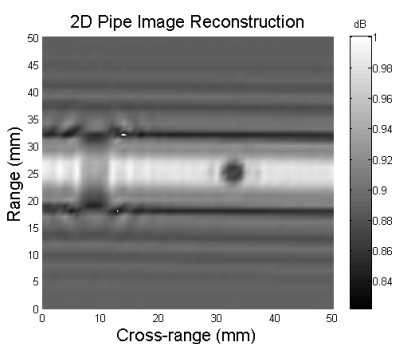

(b)

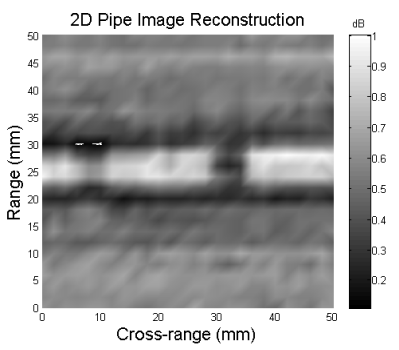

(f)

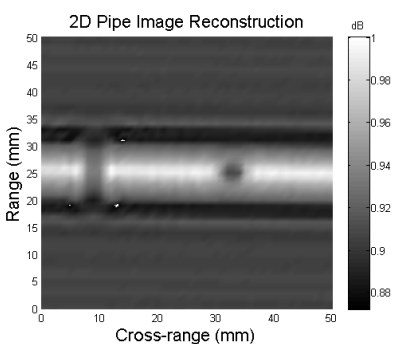

(c)

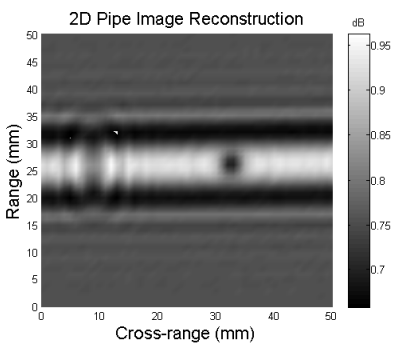

(g)

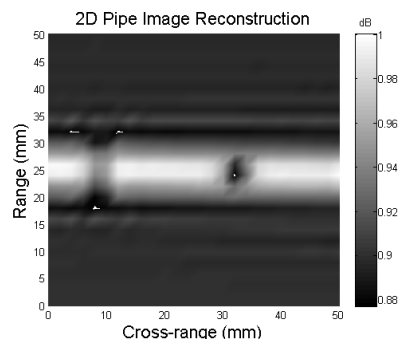

(d)

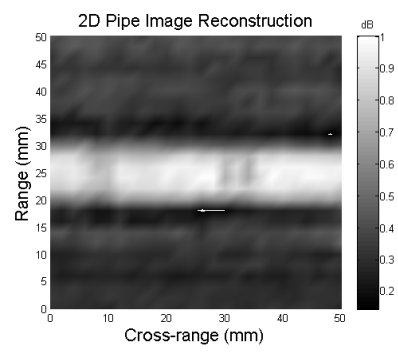

(h)

Fig. 6. Reconstructed pipe images (a) RDA at $d=40 \mathrm{~mm}$, (b) $\omega-k$ at $d=40 \mathrm{~mm}$, (c) RDA at $d=60 \mathrm{~mm}$, (d) $\omega-k$ at $d=60 \mathrm{~mm}$, (e) RDA at $d=80$ $\mathrm{mm}$, (f) $\omega-k$ at $d=80 \mathrm{~mm}$, (g) RDA at $d=100 \mathrm{~mm}$, (h) $\omega-k$ at $d=100 \mathrm{~mm}$.

To show the strength of the RDA approach over $\omega-k$ algorithm as used in [3] in detecting defects at high standoff distances, the standoff distance is varied from $40 \mathrm{~mm}, 60 \mathrm{~mm}$, $80 \mathrm{~mm}$ and $100 \mathrm{~mm}$ and the resultant images are shown in Fig. 6. Also, since only the pipe is present, the SVD mitigation is not needed in this case. From the images presented, at a lower standoff distance of $40 \mathrm{~mm}$ and $60 \mathrm{~mm}$, both algorithms reconstructed the image of the pipe showing the defects on it. However, RDA gives high-resolution images compared to the $\omega-k$ algorithm. When the standoff distance was increased to $80 \mathrm{~mm}$ and $100 \mathrm{~mm}$, the sharpness/resolution of the image decreases for both algorithms. However, the autofocus RDA still reconstructed the pipe image with the defects areas whereas the defects information were lost for the case of the $\omega-k$ algorithm. This is because the SM interpolation used in the $\omega-k$ algorithm amplifies the noise content and the small motion error in the raw data which add an extra RCMerror in the pipe image owing to the high standoff distance. Whereas, the autofocus RDA, the extracted features of the defect was refocused using RDA residual refocusing. This ensures that the defect information is maintained even at high standoff distances. Therefore, for tiny defects detection and characterisation, RDA will be a better choice compared to the $\omega-k$ algorithm. Moreover, the $\omega-k$ algorithm can be used along with other improvement methods but this normally comes with additional complexity and increase computation time of the algorithm as used in [3]. To further evaluate the quality of the defects on the pipe images at different standoff distances presented in Fig. 6, the SNR and IF of the images were computed for both the RDA and the $\omega-k$ algorithm. The SNR and the IF of the image are computed using

$$
S N R=20 \log _{10}\left|\frac{\mu_{\text {pipe }}}{\sigma_{\text {pipe }}}\right|
$$

$$
I F=10 \log _{10}\left(\frac{D C R_{\text {insulation }}}{D C R_{\text {noinsulation }}}\right)
$$

where $\mu_{\text {pipe }}$ is the mean and $\sigma_{\text {pipe }}$ is the standard deviation of the pipe image, $D C R_{\text {insulation }}$, and $D C R_{\text {noinsulation }}$ are the DCRs of the pipe image with and without the SVD suppression. The DCR of the pipe image can be calculated from

$$
D C R=\frac{P_{c}}{P_{d}} \frac{\sum_{u, y \in \Omega_{d}}\left|\mathbf{I}_{S A R}(u, y)\right|^{2}}{\sum_{u, y \in \Omega_{c}}\left|\mathbf{I}_{S A R}(u, y)\right|^{2}}
$$

where $P_{d}$ and $P_{c}$ are the numbers of pixels in the defect and the clutter regions respectively, $\Omega_{d}$ is the defect region, and $\Omega_{c}$ is the clutter region defined by the entire pipe image but excluding the defect region.

The sensitivity of the defected pipe in relation to the one without defect can be quantified using the SE defined by

$$
E_{S E}=\sum_{m=1}^{M} \sum_{n=1}^{N}\left(I_{S A R}\left(u_{m}, y_{n}\right)-I_{S A R, 0}\left(u_{m}, y_{n}\right)\right)^{2}
$$

where $M$ and $N$ represent the number of pixels around defect area, $I_{S A R}$ and $I_{S A R, 0}$ are the intensities of the image for the pipe with and without defect respectively.

Using (22), (23) and (25), the SNR, IF and SE of the reconstructed pipe images in Fig. 6 were computed and their values at different standoff distances were plotted as shown in Fig. 7. From the figure and for the SNR and IF, it can be observed that lower standoff distances give higher SNR and higher IF for both the RDA and the $\omega-k$ algorithm. The IF values for $\omega-k$ algorithm are higher $12 \mathrm{~dB}$ compared to RDA with $10 \mathrm{~dB}$. This can be attributed to the low noise content in the data and this is where $\omega-k$ algorithm works best, but the IF of RDA is still closer to that of the $\omega-k$ algorithm. As the standoff distance increases, the sharpness of the image as shown by the SNR and the IF values decreases significantly. 


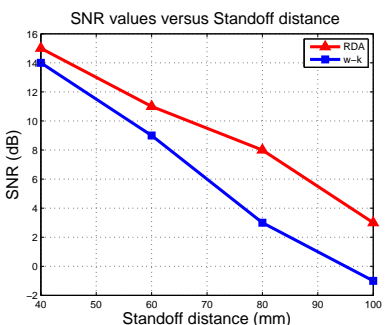

(a)

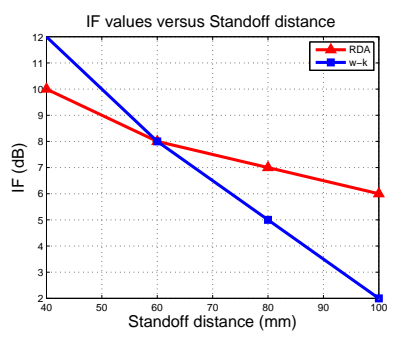

(b)

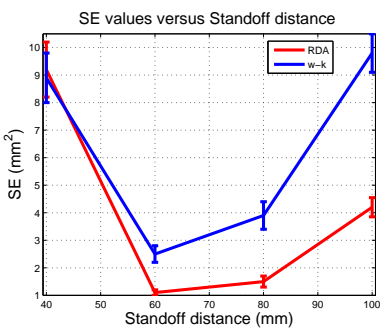

(c)

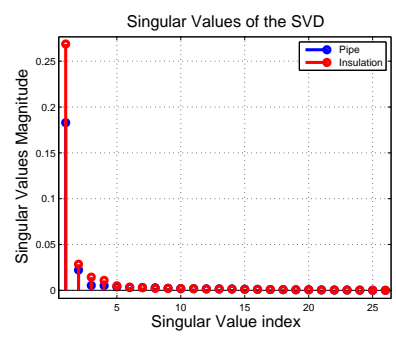

(d)

Fig. 7. Performance evaluation for the pipe images at different standoff distances (a) SNR plot, (b) IF plot, (c) SE plot, (d) SVD of the insulated pipe.

However, the IF values of the RDA is higher than that of the $\omega-k$ algorithm achieving $6 \mathrm{~dB}$ as against $2 \mathrm{~dB}$ for the $\omega-k$ algorithm. This is mainly because the RDA suppressed the noise content of the image better than the $\omega-k$ algorithm. For the case of the SE, at a lower standoff distance of $40 \mathrm{~mm}$, the error in the intensity on the pipe image is relatively high with a value of $9.2 \mathrm{~mm}^{2}$ and $9 \mathrm{~mm}^{2}$ for RDA and $\omega-k$ respectively. This can be attributed to the fact that the sensor is closed to the pipe sample thereby affected by the near-field effect. As the standoff distance increases, the error reduces significantly to a minimum value of $0.1 \mathrm{~mm}^{2}$ for RDA and $0.5 \mathrm{~mm}^{2}$ for $\omega-k$ at a standoff distance of $60 \mathrm{~mm}$. At standoff distance of 80 $\mathrm{mm}$ and above, the error is still less for the RDA with a value of $0.2 \mathrm{~mm}^{2}$, while it suddenly increases for $\omega-k$ to a value of $0.7 \mathrm{~mm}^{2}$ and this is because of the SM error amplification in the $\omega-k$ algorithm. Therefore, for defect detection at higher standoff distance, RDA offers better performance compared to the $\omega-k$ algorithm. When an insulation layer is added, the decrease in SNR and IF values become more pronounced because the standoff distance increases thereby reducing the intensity and sharpness of the image. This effect is studied in the next section.

\section{Validation of the SVD Mitigation for Pipeline Inspection under Insulation Media}

Building on the imaging technique discussed in section III, an insulation layer with a thickness of $40 \mathrm{~mm}$ is used to cover the pipe, and this makes the system a multi-layer problem. The standoff distance from the OEW to the insulation layer is $60 \mathrm{~mm}$ making the total standoff distance to be $100 \mathrm{~mm}$. With the additional layer and standoff distance, the experiment was carried out and the results processed and presented in Fig. 8 for the case of RDA only. The results for the $\omega-k$ algorithm were not shown because the defect information is already lost even without the insulation as shown in Fig. 6 (h). From the pipe image in Fig. 8(a), it can be seen that the defects information are completely lost and only the pipe can be seen from the image. This is because, the reflections from the insulation layer, the high standoff distance, and the ringing effects dominate and obscure the defects on the pipe which make the signal attenuated. To suppress this effect, looking at the SCs of the SVDs of the pipe image shown in Fig. 9, it can be seen that the singular values of the insulation layer are higher than that of the pipe. This is because considering the pipe model system design, since the pipe is insulated, the reflections from the insulation layer are relatively stronger compared to the reflections from the pipe. Therefore,

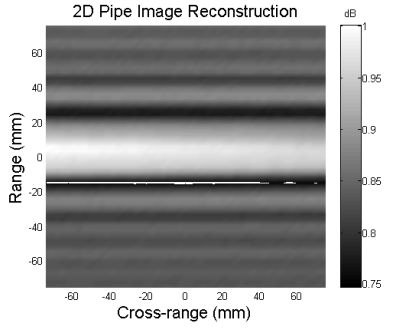

(a)

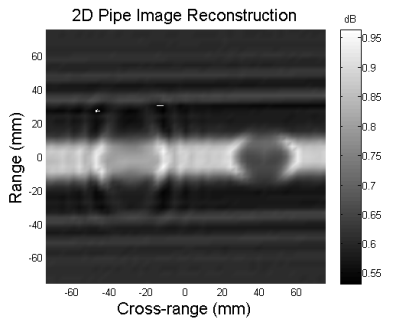

(c)

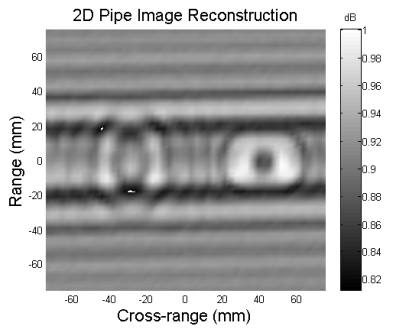

(e)

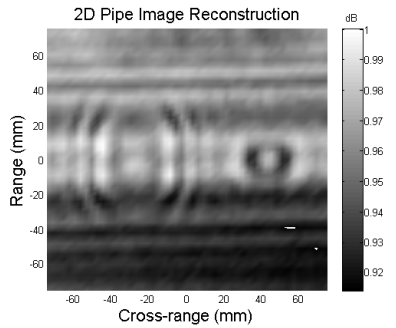

(b)

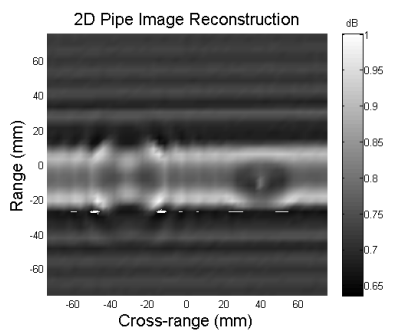

(d)

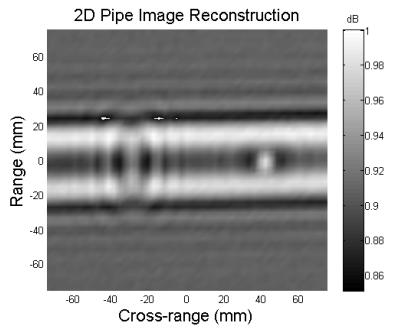

(f)
Fig. 8. Reconstructed images of the defect on the pipe (a) all SCs, (b) suppressing the first SC, (c) suppressing the first 2 SCs, (d) suppressing the first 3 SCs, (e) suppressing the first 4 SCs, (f) suppressing the first 5 SCs.

the insulation layer returns mostly lie in the subspace that is spanned by the singular vectors which characterised the dominant singular values. To suppress its effect, the singular vectors that are associated with the dominant singular values are discarded before forming the image. Hence, the singular vectors that are associated with the dominant singular values are discarded before forming the image. The image in Fig. 8(a) is formed with all the singular values of the image while the images in Fig. 8(b-f) is formed by removing the first, second, third, fourth and fifth singular values. It can be seen that as the singular values are suppressed (removed), the defects on the pipe image becomes visible. The first singular value is dominant among the singular values of the SVD. Therefore 
TABLE I

SNR, IF AND SE VALUES OF THE DEFECT MEASUREMENT FROM THE PIPE IMAGES.

\begin{tabular}{|l|l|l|c|}
\hline Method & SNR $(\mathrm{dB})$ & $\mathrm{IF}(\mathrm{dB})$ & $\mathrm{SE}\left(\mathrm{mm}^{2}\right)$ \\
\hline Non-insulated Pipe & 3 & 6 & 4.2 \\
Insulated pipe & -14 & -12 & 60 \\
First SC removal & -8 & -8 & 35 \\
2nd SCs removal & -4 & -5 & 16 \\
3rd SCs removal & -2 & -1 & 8 \\
4th SCs removal & 0 & 2 & 7 \\
5th SCs removal & 1 & 4 & 6 \\
\hline
\end{tabular}

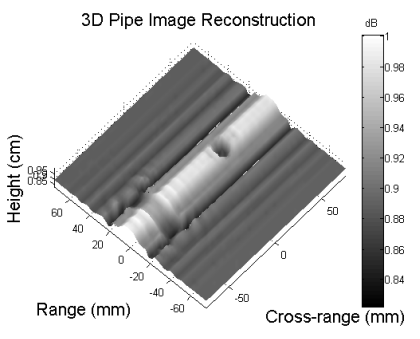

(a)

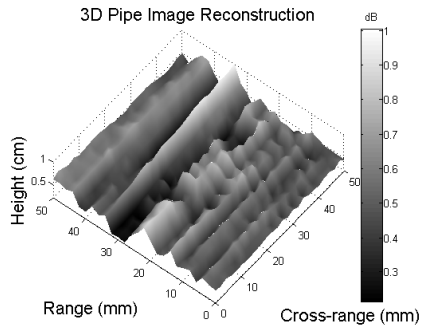

(c)

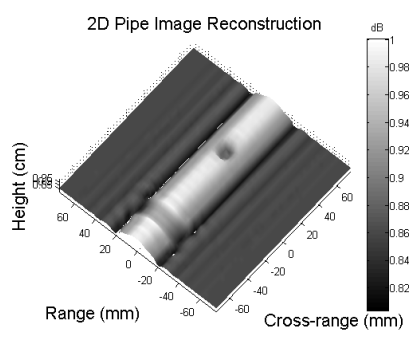

(b)

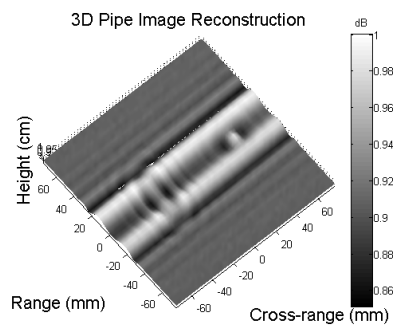

(d)
Fig. 9. 3D pipe image reconstruction (a) SVD- $\omega-k$ without insulation, (b) SVD-RDA without insulation, (c) SVD- $\omega-k$ with insulation, (d) SVD-RDA with insulation.

suppressing it, makes the defect to become visible but the pipe image is still unfocused because of the residuals from the other non-dominant singular values. From the values of the SVDs presented in Fig. 9, the first four singular values are the dominant singular values and that is why after suppressing them, the defect is clearly visible and the image obtained is comparable with the case when no insulation is used. To evaluate the performance of the SVD suppression used in Fig. 8 in comparison to the case without any insulation, the SNR, IF and the SE measurements are used and their computed values are summarised in table I. However, in this case for the SE in (25), $I_{S A R}$ and $I_{S A R, 0}$ stand for the intensities of the image for the insulated and non-insulated pipe respectively. From table I, it can be seen that the reconstructed pipe image without insulation gives the best SNR and IF compared to all cases of the insulated pipe and this is expected because there is no any layer in between the sensor and the pipe. For the case of the insulated pipe with all the SCs used, the IF value is closed to zero because the defect area cannot be seen as a result of the insulation. As the dominant SCs are removed, both the SNR and the IF values increases because the defects area becomes more visible and the case of when the first five SCs are removed achieved comparable performance in relation to the case of the pipe without insulation. For the case of the SE values, as the SCs are removed from first SCs to the 5 th $\mathrm{SCs}$, the values reduce significantly tending towards the value of the case of the pipe without insulation. This shows the effectiveness of the approach in suppressing the effect of the insulation layer. Thus, it can be concluded that using the approach of SVD-RDA, the technique can be used to detect tiny defects on insulated pipes and the results are comparable to the case when no insulation is used.

\section{3D Pipeline Inspection under an Insulation Media}

In 3D imaging of the pipe, the same raster C-scan for the $2 \mathrm{D}$ imaging is used. The 2-D scanning is done along the horizontal and vertical directions to reveal the characteristics of the pipe under the insulation layer. The height of the pipe is added to form the 3-D image. The image using the SVD-RDA approach is formed and for comparison purpose, SVD $-\omega-k$ algorithm image is also formed and the results are shown in Fig. 10. Fig. 10 (a-b) are the images of the pipe without the insulation layer while Fig. 10 (c-d) is the one with the insulation layer. In both cases, the standoff distance used is $100 \mathrm{~mm}$ and from the images presented, it can be seen that for the case when only the pipe was scanned, both approaches reconstructed the pipe image with the SVD-RDA revealing the defects clearer than the SVD- $\omega-k$ approach. For the case of the insulated pipe, the information about pipe curvature and the defects were completed lost for the case of $\omega-k$ algorithm based on the reason explained in section III. For the case of SVD-RDA, the pipe curvature and defects were maintained and can be clearly seen from the image. In terms of performance, the SNR and IF values remain the same as previously computed for the $2 \mathrm{D}$ images, i.e. the SVD-RDA approach gave higher SNRs and IF compared to the $\omega-k$ algorithm for both cases of the insulated and non-insulated pipe. However, the SNR and IF values decrease for the insulated pipe which is expected because of the layer added by the insulation.

To conclude, our approach is compared with the existing state-of-art within the area of microwave imaging and it is summarised in table II. It can be clearly seen that the proposed approach outperformed most of the current methods in terms defect detection and evaluation, mitigating the insulation by improving the SNR, and exhibit superior features at a high standoff distance. However, the approach is slow compared to other methods and this area needs to be improved in future work.

\section{CONCLUSION}

The paper proposed a new technique of autofocus RDA SAR-based microwave imaging for pipeline inspection under an insulated media. Based on the challenges posed by the pipe curvature and high standoff distance introduced by the insulation layer, SVD and autofocus RDA were developed to mitigate their effect and reconstructed the defects area on the pipe. SVD was used to mitigate the effect of the insulation layer by removing the dominant singular values that characterise the insulation layer, and autofocus RDA to refocus the reconstructed pipe image using RDA residual refocusing. 
TABLE II

PROPOSED METHOD COMPARISON WITH STATE-OF-THE-ART IN MICROWAVE IMAGING SYSTEMS.

\begin{tabular}{|c|c|c|c|c|c|c|}
\hline Method & $\begin{array}{l}\text { Operating } \\
\text { frequency }\end{array}$ & $\begin{array}{l}\text { Image } \\
\text { quality }\end{array}$ & $\begin{array}{c}\text { Defect } \\
\text { detection }\end{array}$ & $\begin{array}{c}\text { SNR } \\
\text { improvement }\end{array}$ & $\begin{array}{l}\begin{array}{c}\text { Scanning } \\
\text { distance }\end{array} \\
\end{array}$ & $\begin{array}{l}\text { Computation } \\
\text { time }\end{array}$ \\
\hline PW-SAR and WL-SAR [3] & X-band & $\begin{array}{l}\text { High image } \\
\text { resolution }\end{array}$ & Medium & $\begin{array}{l}\text { Very good at } \\
\text { near field }\end{array}$ & $\begin{array}{l}\text { Very good for } \\
\text { near field }\end{array}$ & Slow \\
\hline Robust adaptive GPR [11] & K-band & $\begin{array}{l}\text { High image } \\
\text { resolution }\end{array}$ & No & $\begin{array}{l}\text { Good at } \\
\text { far field }\end{array}$ & $\begin{array}{l}\text { Good for } \\
\text { far field }\end{array}$ & Slow \\
\hline Subspace projection [21] & $2-3 \mathrm{GHz}$ & $\begin{array}{l}\text { High image } \\
\text { resolution }\end{array}$ & No & $\begin{array}{l}\text { Very good at } \\
\text { far field }\end{array}$ & $\begin{array}{l}\text { Very good for } \\
\text { far field }\end{array}$ & Fast \\
\hline CS-SAR imaging [32] & $2.25 \mathrm{GHz}$ & $\begin{array}{l}\text { High image } \\
\text { resolution }\end{array}$ & No & $\begin{array}{l}\text { Medium at } \\
\text { far field }\end{array}$ & $\begin{array}{l}\text { Good for } \\
\text { far field }\end{array}$ & Fast \\
\hline Proposed approach & K-band & $\begin{array}{l}\text { High image } \\
\text { resolution }\end{array}$ & High & $\begin{array}{l}\text { Very good at } \\
\text { near field and } \\
\text { good for } \\
\text { far field }\end{array}$ & $\begin{array}{l}\text { Very good for } \\
\text { near field and } \\
\text { good for } \\
\text { far field }\end{array}$ & Slow \\
\hline
\end{tabular}

Experimental validation was carried out to show the strength of the RDA technique compared to the well-known $\omega-k$ algorithm in dealing with the pipe curvature and standoff distance. The SNR, IF and SE were used as performance measures to evaluate the performance of the SVD suppression and the qualitative defect information of the reconstructed pipe image. The IF in terms of DCR were used to study the point at which all the dominant singular values were suppressed and used as the point to form the pipe image. It was found that the autofocus RDA gave higher values of SNRs and IFs ( $3 \mathrm{~dB}$ and $5 \mathrm{~dB})$ compared to the $\omega-k$ algorithm $(-1 \mathrm{~dB}$ and $2 \mathrm{~dB})$ respectively. The SE is used to measure the sensitivity of the defect on the pipe, and the results obtained shows that RDA is less sensitive to noise compared to the $\omega-k$ algorithm as the standoff distance increases. This shows the robustness of the autofocus RDA in inspecting inspected pipes at high standoff distance by retaining the defect information and focusing the image.

In future work, the technique will be extended to inspect pipes at far-filed or near-far-field region suitable for inspecting buried pipes underneath the ground. Also, as the signal is sparse, the sparsity can be used to reduce the computation time thereby improving the image resolution of the pipe.

\section{ACKNOWLEDGMENT}

The authors like to acknowledge the support of Newcastle University and Petroleum Trust Development Fund Nigeria for providing sponsorship to the first author.

\section{REFERENCES}

[1] A. Cataldo, G. Cannazza, E. De Benedetto and N. Giaquinto, "A New Method for Detecting Leaks in Underground Water Pipelines," IEEE Sensors J., vol. 12, no. 6, pp. 1660-1667, June 2012.

[2] S. M. O'Connor, J. P. Lynch, M. Pour-Ghaz, S. S. Nadukuru, R. L. Michalowski, R. A. Green, A. Bradshaw and W. Jason Weiss, "Chapter 8 - Underground Sensing Strategies for the Health Assessment of Buried Pipelines," Underground Sensing, Academic Press, pp 417-453, 2018,

[3] M. Fallahpour, J. T. Case, M. T. Ghasr and R. Zoughi, "Piecewise and Wiener Filter-Based SAR Techniques for Monostatic Microwave Imaging of Layered Structures," IEEE Trans. on Antennas and Prop., vol. 62, no. 1, pp. 282-294, Jan. 2014.
[4] E. H. A. Duisterwinkel, E. Talnishnikh, D. Krijnders and H. J. WÃúrtche, "Sensor Motes for the Exploration and Monitoring of Operational Pipelines," IEEE Trans. on Inst. and Meas., vol. 67, no. 3, pp. 655-666, March 2018.

[5] R. E. Malkin, A. C. Franklin, R. T. Bevan, H. Kikura and B. W. Drinkwater,"Surface reconstruction accuracy using ultrasonic arrays: Application to non-destructive testing," NDT\&E Int., vol. 96, pp. 26-34, 2018.

[6] Y. He, G. Tian, H. Zhang, M. Alamin, A. Simm and P. Jackson, "Steel Corrosion Characterization Using Pulsed Eddy Current Systems," IEEE Sensors J., vol. 12, no. 6, pp. 2113-2120, June 2012.

[7] R. Jarvis, P. Cawley and P.B. Nagy, "Current deflection NDE for the inspection and monitoring of pipes," NDT\&E Int., vol. 81, pp. 46-59, 2016.

[8] H. Q. Pham et al., "Highly Sensitive Planar Hall Magnetoresistive Sensor for Magnetic Flux Leakage Pipeline Inspection," IEEE Trans. on Magnet., vol. 54, no. 6, pp. 1-5, June 2018.

[9] J. Feng, F. Li, S. Lu, J. Liu and D. Ma, "Injurious or Noninjurious Defect Identification From MFL Images in Pipeline Inspection Using Convolutional Neural Network," IEEE Trans. on Inst. and Meas., vol. 66, no. 7, pp. 1883-1892, July 2017.

[10] Nurmalia, N. Nakamura, H. Ogi and M. Hirao, "EMAT pipe inspection technique using higher mode torsional guided wave $\mathrm{T}(0,2)$, , $N D T \& E$ Int., vol. 87, pp. 78-84, 2017.

[11] Q. Hoarau, G. Ginolhac, A.M. Atto and J.M. Nicolas, "Robust adaptive detection of buried pipes using GPR," Signal Processing, vol. 132, pp. 293-305, 2017.

[12] M. D. Buhari, G. Y. Tian, R. Tiwari and A. H. Muqaibel, "Multicarrier SAR Image Reconstruction Using Integrated MUSIC-LSE Algorithm," IEEE Access, vol. 6, pp. 22827-22838, 2018.

[13] M. Coramik and Y. Ege,'Discontinuity inspection in pipelines: A comparison review," Measurement, vol. 111, pp. 359-373, 2017.

[14] J. De Zaeytijd, A. Franchois, C. Eyraud and J. M. Geffrin, "FullWave Three-Dimensional Microwave Imaging With a Regularized GaussâĂŞNewton MethodâĂ $\breve{T}$ Theory and Experiment," IEEE Trans. on Antennas and Prop., vol. 55, no. 11, pp. 3279-3292, Nov. 2007.

[15] J. De Zaeytijd, A. Franchois and J. M. Geffrin, "A New Value Picking Regularization StrategyâĂŤApplication to the 3-D Electromagnetic Inverse Scattering Problem," IEEE Trans. on Antennas and Prop., vol. 57, no. 4, pp. 1133-1149, April 2009.

[16] D. W. Winters, B. D. Van Veen and S. C. Hagness, "A Sparsity Regularization Approach to the Electromagnetic Inverse Scattering Problem," IEEE Trans. on Antennas and Prop., vol. 58, no. 1, pp. 145-154, Jan. 2010.

[17] C. Gilmore, I. Jeffrey and J. LoVetri, "Derivation and comparison of SAR and frequency-wavenumber migration within a common inverse scalar wave problem formulation," IEEE Trans. Geosci. Remote Sens., vol. 44, no. 6, pp. 1454-1461, June 2006.

[18] Ankita and A. Basu, "Microwave imaging using distributed sensors," IEEE MTT-S International Microwave and RF Conference, New Delhi, pp. 1-4, 2013. 
[19] F. Boero et al., "Microwave Tomography for the Inspection of Wood Materials: Imaging System and Experimental Results," IEEE Trans. on Microwave Theo. and Techn., vol. 66, no. 7, pp. 3497-3510, July 2018.

[20] L. Ran, Z. Liu, T. Li, R. Xie and L. Zhang, "Extension of Map-Drift Algorithm for Highly Squinted SAR Autofocus," IEEE J. Sel. Topics Appl. Earth Observ. Remote Sens., vol. 10, no. 9, pp. 4032-4044, Sept. 2017.

[21] F. H. C. Tivive, A. Bouzerdoum and M. G. Amin, "An SVD-based approach for mitigating wall reflections in through-the-wall radar imaging," 2011 IEEE RadarCon (RADAR), Kansas City, MO, 2011, pp. 519-524.

[22] F. H. C. Tivive, A. Bouzerdoum and M. G. Amin, "A Subspace Projection Approach for Wall Clutter Mitigation in Through-the-Wall Radar Imaging," IEEE Trans. Geosci. Remote Sens., vol. 53, no. 4, pp. 2108-2122, April 2015.

[23] M. M. Riaz and A. Ghafoor, âĂIJThrough-Wall Image Enhancement Based on Singular Value Decomposition,âĂİ Int. Journ. of Ant. and Prop., vol. 2012, 20 pages, 2012.

[24] D. Garmatyuk and M. Brenneman, "Adaptive Multicarrier OFDM SAR Signal Processing," IEEE Trans. Geosci. Remote Sens., vol. 49, no. 10, pp. 3780-3790, 2011.

[25] D. Feng, D. An and X. Huang, "An Extended Fast Factorized Back Projection Algorithm for Missile-Born Bistatic Forward-Looking SAR Imaging," IEEE Trans. on Aerospace and Elect. Syst.(Early Access).

[26] M. Younis, T. Rommel, F. Bordoni, G. Krieger and A. Moreira, "On the Pulse Extension Loss in Digital Beamforming SAR," IEEE Trans. Geosci. Remote Sens. Lett., vol. 12, no. 7, pp. 1436-1440, July 2015.

[27] J. C. Ni, Q. Zhang, Y. Luo and L. Sun, "Compressed Sensing SAR Imaging Based on Centralized Sparse Representation," IEEE Sensors J., vol. 18, no. 12, pp. 4920-4932, June15, 152018.

[28] J. Yu and H. Liu, "Sparse Coding Shrinkage in Intrinsic Time-Scale Decomposition for Weak Fault Feature Extraction of Bearings," IEEE Trans. on Inst. and Meas., vol. 67, no. 7, pp. 1579-1592, July 2018.

[29] M. Ghorat, G. B. Gharehpetian, H. Latifi and M. A. Hejazi, "A New Partial Discharge Signal Denoising Algorithm Based on Adaptive DualTree Complex Wavelet Transform," IEEE Trans. on Inst. and Meas.(Early Access).

[30] M. D. Buhari, G. Y. Tian, R. Tiwari and A. H. Muqaibel, "OFDM SAR multiple targets image reconstruction using MUSIC-LSE algorithm," 2016 IEEE Int. Work. on Compressed Sensing Theo. and its App. to Radar, Sonar and Remote Sen. (CoSeRa), pp. 42-46, Sep. 2016.

[31] M. D. Buhari and A. H. Muqaibel, "SAR multiple targets imaging using UWB OFDM signals," IEEE 9th Int. Symp. on Comm. Sys., Net. and Digital Signal Process. (CSNDSP), pp. 485-490, Sep. 2014.

[32] M. D. Buhari, G. Y. Tian, R. Tiwari and A. H. Muqaibel, "Adaptive OFDM subcarrier design for SAR Cross-Range Profile Reconstruction," 2016 IEEE Int. Conf. for Stud. on Applied Eng. (ICSAE), pp. 485-490, Oct. 2016.

[33] K. Aberman and Y. C. Eldar, "Sub-Nyquist SAR via Fourier Domain Range-Doppler Processing," IEEE Trans. Geosci. Remote Sens., vol. 55, no. 11, pp. 6228-6244, Nov. 2017.

[34] Wellpack Europe, "Multi Sizes Polyethylene Etha Foam Stratocell/Closed Cell Foam Sheets Blocks UK 2018," Available at: https://www.amazon.co.uk/.

[35] S. Kharkovsky and R. Zoughi, "Microwave and millimeter wave nondestructive testing and evaluation - Overview and recent advances," IEEE Trans. on Inst. and Meas., vol. 10, no. 2, pp. 26-38, April 2007.

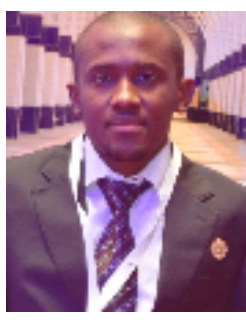

wall radar imaging.

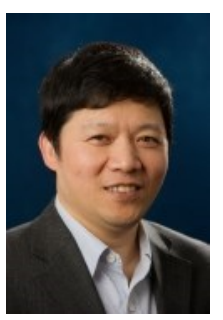

Gui Yun Tian received his B.Sc. degree and M.Sc. degree from University of Sichuan, Chengdu, China in 1985 and 1988, respectively, and Ph.D. from University of Derby, Derby, UK, in 1998. He is currently the Professor of Sensor Technologies at School of Electrical and Electronic Engineering, Newcastle University, United Kingdom. His main interests include Electromagnetic sensors, sensor array and sensor network, Electromagnetic Nondestructive Evaluation, Advanced signal processing and Integrative systems and applications.

He has coordinated several research projects from the Engineering and Physical Sciences research Council (EPSRC), Royal Academy of Engineering and FP7. Also he has good collaboration with leading industrial companies such as Airbus, Rolls Royce, BP, nPower, Network Rail and TWI among others.

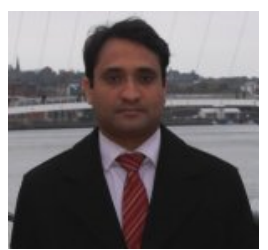

Rajesh Tiwari Ph.D, is Teaching Fellow at School of Electrical and Electronic Engineering, Newcastle University, UK. He has background research in Global Navigation Satellite System (GNSS), channel modelling, wireless location and GNSS software receiver designing and remote sensing. Research interest also includes space weather effect on GPS, he participated as a Scientist in XXVI Indian Scientific Expedition to Antarctica.

In Antarctica, he studied the effect of Aurora on GPS receiver and also did several experiments in marine navigation using GPS receiver, the results published in a book chapter title "Antarctica: The Most Interactive Ice-Air-Ocean Environment". He received various prestigious award over the period; Young Scienctist Award, Indian Science Congress, Bhopal Chapter, India, Young Scientist Award (2011), International Union of Radio Science. He published 15 journal papers, and PI of EPSRC IAA KTS project. 